076

\section{VALIDITÀ DI UN TEST IGG IGM WESTERN-BLOT NELLA DIAGNOSI PRECOCE DELL'INFEZIONE CONGENITA DA T. GONDII}

Meroni V., Genco F., Piccoli L., Bollani L.', Stronati M.'

\section{Dipartimento di Clinica di Malattie Infettive \\ Università degli Studi Pavia, \\ ' Unità Terapia Intensiva Neonatale \\ IRCCS Policlinico San Matteo Pavia \\ Via Taramelli 527100 Pavia}

Introduzione. La diagnosi di toxoplasmosi congenita nel neonato a rischio asintomatico rimane a tutt'oggi un problema insoluto nonostante l'esigenza di una definizione precoce dello stato di infezione: da una parte per trattare tempestivamente i neonati infetti e dall'altra per evitare una terapia lunga e poco maneggevole a neonati non infetti. Scopo di questo studio è stato di valutare l'accuratezza diagnostica del test IgG IgM Western-blot (IgG IgM-WB LDBIO Lyon France) in uso nel nostro laboratorio da diversi anni.

Metodi. Sono stati valutati retrosopettivamente con il test IgG IgM WB (LDBIO Lyon France) 224 neonati a rischio: il campione ottenuto alla nascita in parallelo con quello materno e poi con i campioni ottenuti nei primi tre mesi di vita. Tutti i campioni sono stati analizzati in precedenza con i tests in uso nel laboratorio: ELISA IgG IgM, IgA(Diasorin Saluggia Italia), IgG ELFA,Toxo IgM ISAGA (Biomerieux Marcy L'Etoile France). La diagnosi di infezione è stata fatta alla nascita in 30 neonati per la presenza di $\operatorname{IgM}$ e/o $\operatorname{IgA}$, per i restanti 10 sulla base di un rebound anticorpale o per la positività delle IgG all'anno di vita.

Risultati.

\begin{tabular}{lccc}
\hline & Isaga IgM+ELISA & WB IgG IgM & Tot \\
\hline Non infetti & 180 & 177 & 184 \\
\hline Infetti & 30 & 38 & 40 \\
\hline Sensibilità & $75 \%$ & $96 \%$ & \\
\hline Specificità & $98 \%$ & $96 \%$ & \\
\hline
\end{tabular}

Conclusioni. Il test in esame ci ha permesso di trattare tempestivamente (nei primi tre mesi di vita) 8 neonati infetti negativi con $i$ tests tradizionali e di non trattare 177 neonati non infetti.

\section{7}

\section{EPIDEMIOLOGIA DELLE PARASSITOSI INTESTINALI A PARMA NELL'ANNO 2005.}

\author{
Calderaro A., Gorrini C., Peruzzi S., Piccolo G..,
} Bommezzadri S., Dettori G.., Chezzi C.

Dipartimento di Patologia e Medicina di Laboratorio, Sezione di Microbiologia, Università degli Studi di Parma.

Introduzione. Le parassitosi intestinali rappresentano un rilevante problema di salute nei Paesi in via di sviluppo, mentre nei Paesi industrializzati come l'Italia la loro epidemiologia è poco nota. In questo studio riportiamo i casi di parassitosi intestinali osservati a Parma durante l'anno 2005. Metodi. Sono stati analizzati campioni di feci di 1117 pazienti (166 stranieri e 951 italiani) pervenuti al nostro laboratorio da reparti ospedalieri e da ambulatori distribuiti sul territorio. Ciascun campione è stato sottoposto ad esame macroscopico per evidenziare consistenza, presenza di sangue, muco e/o forme adulte di elminti e ad esame microscopico per la ricerca di cisti e/o trofozoiti di protozoi e uova e/o larve di elminti. Nei campioni in esame sono stati anche ricercati antigeni di Giardia intestinalis e Cryptosporidium spp., protozoi ed elminti mediante esame colturale e, quando necessario, Entamoeba histolytica e E. dispar mediante PCR specifica.

Risultati. Sono stati diagnosticati 146 casi $(13,07 \%)$ di infezioni da parassiti intestinali (95 in soggetti italiani, 51 in stranieri; 19 bambini, 127 adulti)

112 erano infezioni singole causate da protozoi

(87 Blastocystis hominis e 18 Giardia intestinalis), ed elminti (3 Strongyloides stercoralis,

2 Taenia saginata,

1 Enterobius vermicularis,

1 Ascaris lumbricoides).

34 erano infezioni miste: 7 B.hominis + Entamoeba coli,

4 G.intestinalis + B.hominis,

4 S.stercoralis + B.hominis,

2 E.vermicularis + B.hominis,

1 S.stercoralis + B.hominis + Entamoeba coli,

1 T.trichiura + G.intestinalis + H.nana + B.hominis +

Entamoeba coli,

1 Taenia spp.+ Diphyllobotrium latum,

1 E. histolytica + B.hominis,

1 Acanthamoeba spp. +B.hominis,

1 E.dispar+B.hominis +Entamoeba coli,

1 D.fragilis $+B$.hominis,

1 D. fragilis + B.hominis + Entamoeba coli,

2 G.intestinalis + H.nana + E.dispar + B.hominis,

1 G.intestinalis + E.dispar + B.hominis,

1 S.stercoralis + H.nana + B.hominis + Entamoeba coli,

1 A.duodenale + T.trichiura + B.hominis,

1 E. histolytica + A.lumbricoides,

1 G.intestinalis + Taenia spp. + B.hominis,

1 Taenia spp. + B.hominis,

1 Taenia saginata + B.hominis.

Conclusioni. Solo grazie all'esecuzione di un esame parassitologico completo (indagini tradizionali e molecolari) è stato possibile evidenziare che le parassitosi intestinali rappresentano una realtà epidemiologica rilevante nella nostra area, dove le precarie condizioni igieniche alimentari e/o l'immigrazione rivestire un ruolo importante. 